\title{
Ant Colony Optimization Based on Combined Optimization for Path Planning
}

\author{
Bin Ge \\ School of Information Engineering, Dalian University \\ Dalian University \\ Dalian, China \\ gebin58@163.com
}

\author{
Houyuan Sheng \\ School of Information Engineering, Dalian University \\ Dalian University \\ Dalian, China \\ shenghouyuan@163.com
}

\begin{abstract}
In order to solve disadvantages of the ant colony algorithm in path planning of mobile robot, an enhanced ant colony optimization based on combined optimization is proposed. Improved ant colony algorithm' idea is selecting the searched full path to combined optimize with the rest of the path when all ants in a generation have completed a path search. That is choosing the shorter road segments of between two paths crossing point to combine into a better path, to produce more of the full path for the global pheromone updating and accelerate the positive feedback effect of pheromone. Experimental results show that the new algorithm is correct and effective.
\end{abstract}

Keywords-ant colony optimization; mobile robot; path planning; combined optimization

\section{INTRODUCTION}

The planning of mobile robots is that the robot in an obstructed environment search an optimal path according to one or more rules (such as minimum energy consumption, the shortest path). The optimal obstacleavoidance path is from the starting point to goal point.

Ant colony algorithm has many advantages like information positive feedback effect, excellent parallel computing, strong ability of heuristic searching, etc. But ant colony algorithm has many disadvantages like lack of initial pheromone, solving slowly, being easy falling into local optimal solution. Improved ant colony algorithm idea is selecting the searched full path to combined optimize with the rest of the path when all ants in a generation have completed a path search. That is choosing the shorter road segments of between two paths crossing point to combine into a better path to replace searched path and update global pheromone. This can speed up information positive feedback effect and increase the diversity of solution.

\section{ENVIRONMENT MODELING OF MOBILE ROBOT}

The grid map is easy to create and modify. Information of grid zone corresponds directly with the real environment, so the self-localization and path planning is easy. Hence, the grid method is used to environmental modeling.

For any grid $g$, if it is occupied partially or fully by an obstacle, it is called obstacle grid to robots do not pass and is expressed in 1; otherwise, $\mathrm{g}$ is labeled as free grid to robots can freely pass and is expressed in 0 . The size of the grid need to be moderate. The smaller the grid is, the more precise the obstacles are described. But this takes up too much storage space and will increase exponentially the algorithm' search volume. On the other hand, the planning path will be less accurate when the grid is too large. The size of the grid is consistent with robot's the movement length. Namely robot each can only move from one grid to a neighboring grid. In this paper, all grids have different sequence numbers. When size $\mathrm{N}=6$ of the environment model, which is shown in Fig. 1.

As it is shown in Fig. 1, any grid g's rectangular coordinate (xi,yi) and sequence numbers $i$ are mapping relation.The coordinate values of serial number $i$ is calculated by the following formula, where "mod" demotes modulo operation and "fix" demotes rounding operation.

$$
\begin{aligned}
x_{i} & =\left\{\begin{array}{cl}
N & \text { if } \bmod (i, N)=0 \\
\bmod (i, N) & \text { else }
\end{array}\right. \\
y_{i} & =f i x(i, N)+1
\end{aligned}
$$

The coordinate values is used to calculate the linear distance between any two grids. The linear distance is used to calculate distance heuristic function. The serial number $\mathrm{i}$ is calculated by the following formula. The sequence numbers method is used in path representation and path searching.

$$
i=\left(y_{i}-1\right) * N+x_{i}
$$

Figure 1. Model of the grid map 


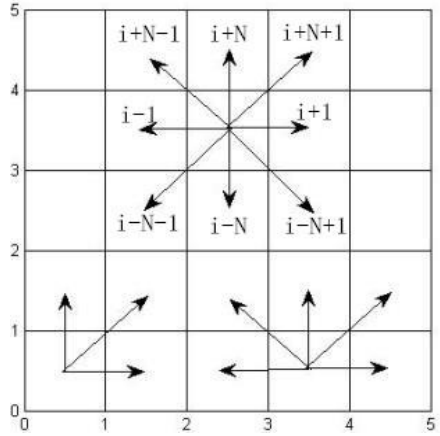

Figure 2. Selecting direction

According to the grid position is different, they are divided into two categories, middle grid and boundary grid. Under the condition without considering the obstacles, the middle grid can choose 8 directions and the boundary grid is far less than 8 directions, as it is shown in Fig. 2. For the convenience of operation, add a circle grid around the environment model.These grids are all obstacle grid.This original grids of the environment model are all middle grid and can choose 8 directions. If ant $m$ locates on current node $\mathrm{i}, \mathrm{LI}$ is the neighbor set.

$L I=[i-1, i+N-1, i+N, i+N+1, i-1, i-N+1, i-N, i-N-1]$

As it is shown in Fig. 2, in order to prevent from the generated path walked through obstacles grid vertex, the following constraints should be added. If the node i- 1 is obstacle grid, the node $\mathrm{i}+\mathrm{N}-1$ and the node $\mathrm{i}-\mathrm{N}-1$ is not selected.If the node $\mathrm{i}-\mathrm{N}$ is obstacle grid, the node $\mathrm{i}+\mathrm{N}-1$ and the node $\mathrm{i}+\mathrm{N}+1$ is not selected.If the node $\mathrm{i}+1$ is obstacle grid, the node $\mathrm{i}+\mathrm{N}+1$ and the node $\mathrm{i}-\mathrm{N}+1$ is not selected.If the node $\mathrm{i}-\mathrm{N}$ is obstacle grid, the node $\mathrm{i}-\mathrm{N}+1$ and the node $\mathrm{i}-\mathrm{N}-1$ is not selected.

Ant in the path searching process is easy to fall into the trap of concave obstacles. So ant will not to continue to search path. Therefore, firstly, it is needed to convex environment model, filling concave obstacle traps,removing some free grid that they could not have become a part of the optimal path, reducing the time of the path search.
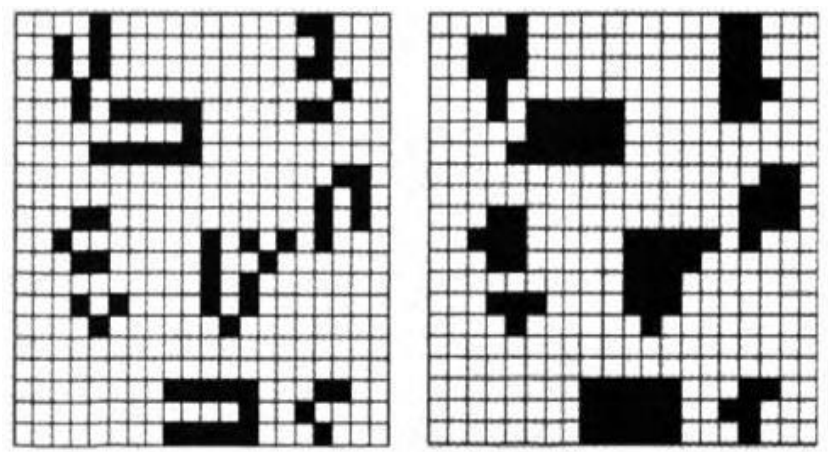

Figure 3. Before the convex

\section{ThE COMBINATION OPTIMIZATION METHOD OF PATH}

In earlier time, probability of the traditional ant colony algorithm is very low to find a path from the starting point to the target point. Especially in complex environment, sometimes just a few or even no ants search out a complete path in each generation, making pheromone positive feedback mechanism cannot take effect. So ants consume a lot of time looking for the full path, rather than on optimizing the complete path .

To solve this problem, this paper puts forward the searched path combination optimization, making full use of the ant searched path to create a more complete path. Then the paper chooses the most I of the combination optimal paths for updating global pheromone. Assuming a complete path is defined as P1 and another unknown path is defined as P2, the combination optimization can be divided into three cases.

Case a): If path P1 and P2 has only one crossing point, new path not is generated.

Case b): If path $\mathrm{P} 1$ and $\mathrm{P} 2$ has more than one crossing point and $\mathrm{P} 2$ is incomplete path, a new combination path consis ts of the shorter paths of P1 and P2 between crossing point and the last part of $\mathrm{P} 1$ path.

Case c): If path P2 is the complete path, a new path is combination of the shorter paths of P1 and P2 between crossing points.

Using this method, it can be seen that it is also worthwhile to making use of the incomplete path.By a combination optimization, it is possible to find a path which is better than the full qualified path. The time spend on a path combination optimization is much shorter than an ant's path searching time, and the chance of the path which is found by combination optimization is far too much bigger than ant at random. Thus, it can be swiftly planed more paths that are from the starting point to the target point. Maybe these fast planned paths are not ideal. But they are complete path, only if more pheromones could be added on these paths, it has an effect on guiding the ant's search and the ant will spend more time in optimizing the complete path.

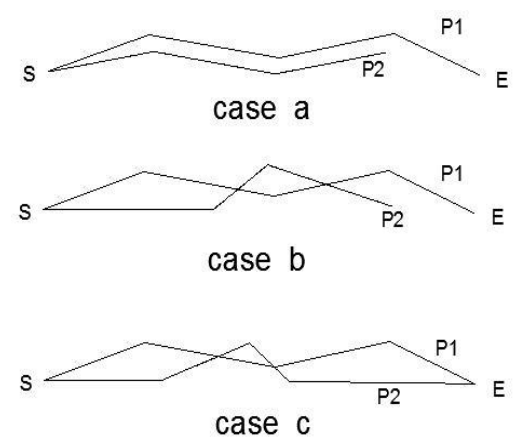

Figure 5. The path combination optimization

Figure 4. After the convex 


\section{ENHANCED ANT COLONY OPTIMIZATION ALGORITHM}

\section{A. The Basic Principle of Algorithmand the Problem Definition}

A kind of material called pheromone can be left on the passed path during the ants' foraging. The ants can sense the strength of the pheromone and tend to move to the place where the concentration of pheromone is high. In this way can they convey the information. The shorter the path is and during the same time, the more ants pass, then the higher concentration of pheromone is and the bigger chance of the ants who come latter choosing this path is. Vice versa, it is the mechanism of pheromone that plays a positive role in foraging. Ant colony algorithm is the bionic algorithm that imitates the behavior of ants' foraging. The starting point of the path planning is regarded as ants' nest location and the target point is regarded as the food's location. Thus the path planning of a moving robot can be regarded as the process that ants starting from their nest, looking for food in the model environment of robot, then through a great number of ants' repetitive foraging, a best path could be found finally.

For the convenience of description, give the following definitions:

Definition 1: $\mathrm{K}$ is number of the evolution generation.M is the number of ants.

Definition 2: $\tau \mathrm{ij}(\mathrm{t})$ is that ants release pheromone on $\operatorname{road}\langle\mathrm{i}, \mathrm{j}\rangle$ at $\mathrm{t}$.

Definition 3. $\Delta \tau i \mathrm{ij}(\mathrm{t})$ is that the ant $\mathrm{m}$ releases pheromone on road $\langle i, j\rangle$ at $t$.

Definition 4. $i$ is the current node number. $j$ is the next node number.LI is the neighbor set of $\mathrm{i}$.

$\mathrm{LI}=[\mathrm{i}-1, \mathrm{i}+\mathrm{N}-1, \mathrm{i}+\mathrm{N}, \mathrm{i}+\mathrm{N}+1, \mathrm{i}-1, \mathrm{i}-\mathrm{N}+1, \mathrm{i}-\mathrm{N}, \mathrm{i}-\mathrm{N}-1]$.

$\mathrm{j}$ is included in LI. Introduce a set ZAD to record all obstacle grids during search procedure. Introduce a set ROUTE to record that the current ant have walked the path point.

Define 5: The distance of between any two grids is calculated by (4).

$$
d(i, j)=\sqrt{\left(x_{i}-x_{j}\right)^{2}+\left(y_{i}-y_{j}\right)^{2}}
$$

The distance heuristic function ,ant $m$ choosing grid $\mathrm{j}$, is determined by Formula (5), where $\mathrm{Q}$ is a large positive constant value.

$$
\eta_{i j}=\frac{Q}{d(j, E)}
$$

Define 6: $\mathrm{L}$ is the length of the ant searching the path ROUTE. Step is the number of the path ROUTE' s points.

$$
L=\sum_{k=1}^{\text {step }-1} d(\operatorname{ROUTE}(k), \quad \operatorname{ROUTE}(k+1))
$$

\section{B. Algorithm Description}

The process of an improved ant colony algorithm is as follows

According to the above definitions, the procedure of enhancement ant colony algorithm is implemented as follow.

Step1: Initializing parameters of the ant colony algorithm used in the process of operation.Let $\mathrm{k}=1, \mathrm{~m}=1$.
Step2: If $\mathrm{k}<=\mathrm{K}$, that is not the number of iterations, go to step3. If $\mathrm{k}>\mathrm{K}$, the algorithm is over and go to step 10 .

Step3: Place the ant $\mathrm{m}$ at the starting point $\mathrm{S}$.The current node number is assigned to i. Put point $S$ in the path node number matrix ROUTE.KXW is the reachable neighbor set,

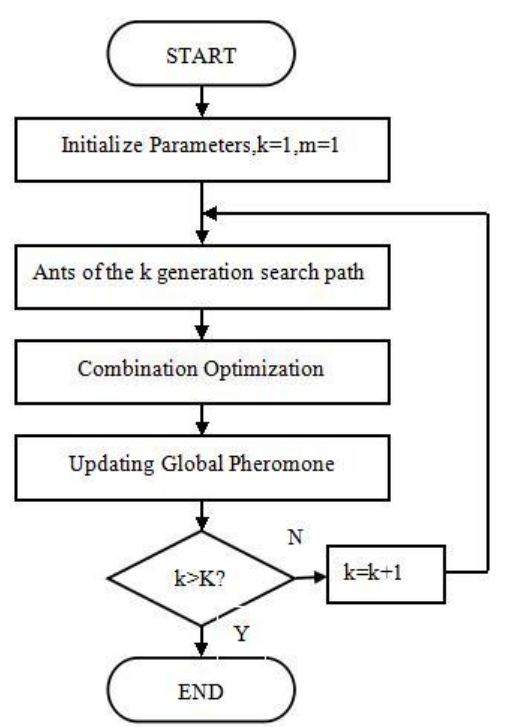

Figure 6. The algorithm flow chart

which in the neighborhood of grid $\mathrm{i}$ and not on the current path and not belongs to the obstacle grids matrix ZAD.

$$
K X W=\operatorname{setdiff}\left(L I,\left[Z A D^{\prime}, R O U T E\right]\right)
$$

Step4: If $\mathrm{i} \neq \mathrm{E}$ and matrix $\mathrm{KXW}$ is not empty,continue to look for the next path point $\mathrm{j}$, otherwise, go to Step 6 . State transition probability, the ant m choosing node $\mathrm{j}$, is calculated by (8), $\alpha$ is the relative importance of pheromone. $\beta$ is the relative importance of distance heuristic function.Selecting the next path points by roulette wheel selection, then the node number is assigned to $\mathrm{j}$.

$$
P_{i j}^{k}=\frac{\tau_{i j}^{\alpha *} \eta_{i j}^{\beta}}{\sum_{s \in K X W} \tau_{i s}^{\alpha} * \eta_{i s}^{\beta}}
$$

Step5: Add the node i to the path matrix ROUTE. Let $\mathrm{i}=\mathrm{j}$. Find out that the next step can choose the reachable set KXW, go to step4.

Step6: Save the path matrix ROUTE to unit array ROUTES $\{k, m\}$. Let $m=m+1$. If $m<=M$, then go to step3.Otherwise go to step7.

Step7: Select the searched full paths to combined optimize with the rest of the path when all the ants in $\mathrm{k}$ generation have completed a path search. Save new paths to the cell array $\mathrm{ZH}$.

Step8: Sorting the new paths, choosing at most I the most optimal path is used to update global pheromone. Updated formula is as follows, where $\rho$ is the pheromone 
evaporate coefficient in the range $(0,1)$ and 1 is length of the path.

$$
\begin{aligned}
& \tau_{i j}=\rho * \tau_{i j}+\Delta \tau_{i j}^{n} \\
& \Delta \tau_{i j}^{n}=Q /
\end{aligned}
$$

Step9: Let $\mathrm{k}=\mathrm{k}+1, \mathrm{~m}=1$.Go to step2.

Step10: End, output the shortest path.

\section{The Simulation TeSt AND PERFORMANCE ANALYSIS}

In order to verify the correctness and validity of the algorithm, simulation experiments are done on the MATLAB platform. Under two complex environments, the basic ant colony algorithm and the improved ant colony algorithm does the contrast experiment. The best optimal path of the basic ant colony algorithm is expressed by the dotted line. The best optimal path of the improved ant colony algorithm is expressed by the solid line. Under two complex environments, the optimal paths are illustrated in Fig. 7 and Fig. 8 respectively.

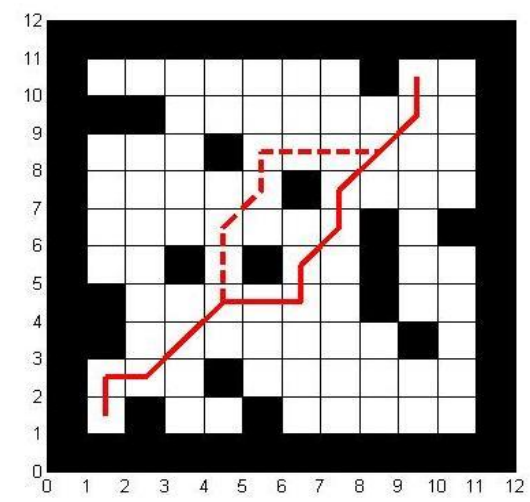

Figure 7. Result under the environment 1

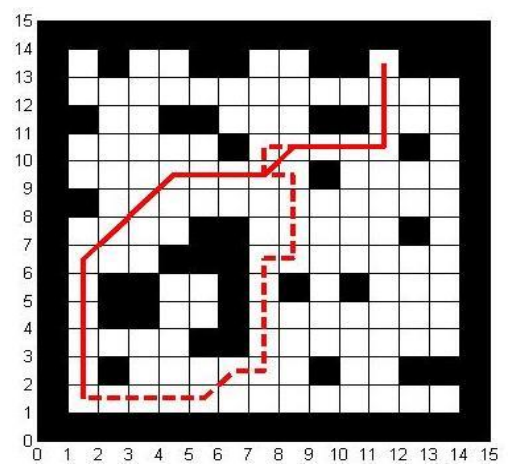

Figure 8. Result under the environment 2
TABLE I. ALGORITHM PERFORMANCE STATISTICS

\begin{tabular}{|c|c|c|c|c|}
\hline Environment & \multicolumn{2}{|c|}{ Environment 1 } & \multicolumn{2}{c|}{ Environment 2 } \\
\hline Algorithm & $\begin{array}{c}\text { Basic } \\
\text { algorithm }\end{array}$ & $\begin{array}{c}\text { New } \\
\text { algorithm }\end{array}$ & $\begin{array}{c}\text { Basic } \\
\text { algorithm }\end{array}$ & $\begin{array}{c}\text { New } \\
\text { algorithm }\end{array}$ \\
\hline Average length & 14.071 & 13.656 & 23.412 & 19.656 \\
\hline Average time & $5.761 \mathrm{~s}$ & $3.243 \mathrm{~s}$ & $7.132 \mathrm{~s}$ & $5.761 \mathrm{~s}$ \\
\hline
\end{tabular}

It can be seen from the table above, the improved algorithm generates the shorter path than the basic ant colony algorithm, taking less time.The improved algorithm is correct and effective.

\section{CONCLUSION}

Based on the existing research results, an improved ant colony algorithm is proposed. The idea is selecting the searched full path to combined optimize with the rest of the path when all ants in a generation have completed a path search, making use of the ant searched path to get more better full path, accelerating the positive feedback effect of pheromone. Simulation experiments show that the planning path and convergence rate are satisfactory.

\section{REFERENCES}

[1] Li Shi-yong.Ant colony algorithm with applications.China:Harbin Institute of Technology press,2004.

[2] CHEN Xiong,ZHAO Yi-lu,HAN Jian-da.An improved ant colony opt imization algorithm for robotic path planning[J].Control Theory Applications, 2010,27(6):821-825.

[3] MA Guan-jun,DUAN Hai-bin,LIU Sen-qi.UCAV Path Planning Based on MAX-MIN Self-adaptive Ant Colony Optimization[J].Acta Aeronautica et Astronautica Sinica, 2008,29(1):243-248.

[4] ZHAO Juan-ping,GAO Xian-wen,FU Xiu-hui,et.al.Improved ant colony algorithm of path planning for mobile robot[J].Control Theory Applications, 201 1,28(4):457-461.

[5] MA Zhan-chun,HAN Yang.Path planning algorithm and simulation for mobile robot[J].Computer Simulation, 2013,30(5):374-378.

[6] WANG Xian,WANG Wei,SONG Shu-lin,et.al.Robot path planning based on ant colony optimization and particle swarm optimization[J],Computer Systems and Applications,2011,20(9):98-102.

[7] FAN Xiao-ping,LUO Xiong,YI Sheng,et.al.Path planning for robots based on ant colony optimization algorithm under complex environment[J].Control and Decision,2004,19(2):166-170.

[8] HUA Lu.Based on ant colony algorithm for path planning research[D].Nanchang:Nanchang Hangkong University, 2011.

[9] LIU Xu-dong.Research and application on the hybrid colony algorithm[D].Anhui:Anhui University of Science and Technology,2013.

[10] LI Jin.Robot path planning research based on ant colony and genetic algorithm[D].Harbin:Harbin Institute of technology,2012. 